\title{
Investigating readiness in the Iranian steel industry through six sigma combined with fuzzy delphi and fuzzy DANP
}

\author{
Peyman Barzegar Keliji ${ }^{*}$, Bahram Shabani Diz Abadi ${ }^{a}$ and Mostafa Abedini ${ }^{a}$
}

${ }^{a}$ Faculty member of Farvardin University, Ghaemshahr, Iran

\begin{tabular}{l}
\hline C H R O N I C L E \\
\hline Article history: \\
Received November 16, 2017 \\
Received in revised format: \\
December 20, 2017 \\
Accepted January 4, 2018 \\
Available online \\
January 5, 2018 \\
\hline Keywords: \\
Six sigma \\
Fuzzy delphi \\
Fuzzy analytic network process \\
Fuzzy DEMATEL \\
Tabarestan steel
\end{tabular}

\section{Introduction}

The economic conditions in which businesses compete today require organizations and management systems to urgently rely on tools that can improve quality and adapt productions/services to rapidly developing situations. Many leading theorists and managers have specifically focused on the issue of quality in such demanding circumstances. Managers are usually confronted with critical conditions such as a shortage of capital, the need to cut costs, and the need to sell more products; in fact, an organization has to be directed in a changing and unstable economic situation. As a qualitative method, the Six Sigma method can have a positive effect on quality, cost reduction, and customer satisfaction, as well as the current leadership of major organizations worldwide (Salehi Sedghiani \& Roustai, 2005). Most of the organizations that have successfully implemented Six Sigma deployments have devised a six-year plan. However, companies can benefit from these projects shortly after the start of the project

* Corresponding author.

E-mail address: peyman.barzegar2016@gmail.com (P. Barzegar Keliji)

(C) 2018 by the authors; licensee Growing Science, Canada. doi: $10.5267 /$ j.dsl.2018.1.001 
if appropriate arrangements are made (NikFarjam \& Norosana, 2009). The implementation of Six Sigma will help improve and maintain quality, eliminate waste, and increase profits (Rajkumar, 2014). Organizations must primarily recognize sources of change and then identify the causes of their emergence, to achieve the desired quality and to be able to lead product quality changes in the right direction. Although these changes may be great in number, it would be more plausible to focus on the most important and determinant factors (Khobyari, 2003). A lack of readiness for the implementation of Six Sigma will result in a sense of disappointment in employees, resistance and opposition (individual, organizational or even political) at different levels, and a tension that could hinder the continuous improvement process (Antony, 2014). Chandra (2008) believes that one of the reasons behind the failure of Six Sigma programs is a lack of proper implementation (Zgardy et al., 2010). Obviously, a model that can assess companies' readiness for Six Sigma deployment can considerably help managers in their decisions. Six Sigma programs have been widely appreciated and conducted in Iranian companies. As the background shows (e.g. Kazazi \& Saroukhani, 2005), the use of Six Sigma programs in the Iranian industrial culture has been very effective, although the dynamics, patterns and mechanisms of Six Sigma projects in such companies remain issues to be further investigated.

The purpose of this study is to probe into the implementation of Six Sigma in an empirical context, Tabarestan Steel Co., in Iran. Besides shedding a new light on the practical aspects of Six Sigma projects, the study can guide Iranian managers on how to make better and more informed decisions about effective deployment of Six Sigma programs, with a view of their organizational constraints. The findings can inspire improvements in the implementation of Six Sigma, and can practically help remove many concerns of senior executives of Tabarestan Steel Co. As mentioned above, an evaluative procedure before fully investing in Six Sigma projects can provide a blueprint of the situation and show how prepared a company is for such projects. This research seeks to (a). design a model for Six Sigma deployment in companies, through the data gathered from Tabarestan Steel Co.; and (b) ultimately formulate a general guideline for consultants and strategists who need to assess the readiness of a company prior to investment in and activation of Six Sigma projects.

\section{Theoretical Foundations}

\subsection{The Notion of Six Sigma}

Six Sigma is a systematic and powerful methodology that uses various tools to improve the quality of processes, products and services (Norosana et al., 2013). Experts dealing with Six Sigma have made efforts to achieve a comprehensive and complete definition of this approach. In this section, some of the most important definitions of the notion are reviewed (Azar et al., 2008). Fogel defines Six Sigma as a clever blend of organizational knowledge, coupled with efficient statistical techniques to improve an organization's efficiency/effectiveness and to meet the real needs of clients (Azar et al., 2008). Mattis contends that the Six Sigma approach tends to accelerate organizational processes that are directed toward goals. This methodology can be used to formulate a set of objectives in the organization (ibid.). (a) encouraging team thinking; (b) creating skills and expertise; (c) making decisions based on facts; (d) making continuous measurement of results and making comparisons with formal organizations; and (e) securing the commitment of senior management. Six Sigma focuses on achieving the goals and criteria and the use of management systems which have to successfully complete projects and sustain long-term profitability. From a technical perspective, Six Sigma concentrates on performance improvement through process data and the use of statistical methods of improvement (Snee, 2000). Six Sigma traditionally tries to reduce costs, and studies have demonstrated that it can also serve as a methodology for increasing profit (Sodhi \& Sodhi, 2005), improving quality (Chen \& Lyu, 2009), stimulating creativity (Biedry, 2001), and boosting organizational learning and facilitating innovation (Byrne et al., 2007; El Haouzi et al., 2009) (see also Krueger et al., 2013). Six Sigma, then, seeks to continuously enhance the improvement process (Lagrosen et al., 2011). 


\subsection{Studies Concerned with Six Sigma}

Henderson and Evans (2000) in a research into the implementation of Six Sigma in General Electric, explored the Six Sigma concept, its benefits, and successful approaches to implementing Six Sigma, especially the operations of General Electric as a pioneering company which introduced the whole notion. Henderson and Evans concluded that the key to success of the Six Sigma was the support and involvement of senior management, organizational structure, training, tools, and relationships with human resource operations.

Hensley and Dobie (2005) evaluated Six Sigma in services, developing a model to help service organizations assess their readiness for Six Sigma programs and to offer suggestions for introducing Six Sigma processes. In their study, two main indicators of organizational readiness were primarily identified through an exploration of the literature, and then an organizational preparedness framework was devised. Chakravorty (2009) addressed the implementation of Six Sigma in the US network technology services, constructing an effective implementation model through the Six Sigma program in a network technology company. This model consists of six steps. (a) conduct a strategic analysis into customers and the market; (b) establish a high-level team aimed at moving toward a primary improvement; (c) identify general improvement tools; (d) display the process map and prioritize improvement opportunities; (e) develop a detailed plan for low-level improvement teams; and (f) make implementation, documentation and revision. The first four phases include strategic decisions that fit into a top-down approach in which management is primarily involved in decision-making. The last two steps involve tactical decisions and follow a bottom-up approach in which engineers and technicians are involved in decision-making from the outset.

Lee et al. (2011) investigated Six-Sigma readiness through a self-assessment model for Chinese companies. They suggested that the main challenge for a successful Six Sigma implementation was readiness. Through this method, organizations could learn about their potentials and flaws in the implementation of Six Sigma. Lagrosen et al. (2011), investigating organizational learning and assessment of Six Sigma deployment preparedness, concluded that organizational learning could provide a framework for assessing the readiness of Six Sigma in an IT organization through a hybrid model they proposed. Probing into preparedness in the Lean Six Sigma (LSS) process in the Higher Education, Antony (2014) described the key factors for implementing Six Sigma. Anthony observed that a number of papers focused on Six Sigma literature to detect the importance of key success factors. The key factors were the essential components that increased the likelihood of success of any initial improvement project before the organization invested its resources. A lack of preparedness would lead to a sense of disappointment in employees, resistance and opposition (individual or organizational or political), and distress which could impede the path to continuous improvement. Finally, Antony concluded that LSS could be a powerful method for controlling process inefficiency in the higher education. Performance Innovation LLC has been working on a team of consultants for over 20 years to provide consultation and training concerned with management and process improvement. With the introduction of Six Sigma, along with leading industry engineering and development of leadership indicators, they help to improve the process. The company operates in the field of pure Six Sigma, leadership improvement, process management, and strategic management. The Six Sigma Readiness Assessment Tool is an effect product; Table 1 reports a summary of the variables for Six Sigma preparation, as observed in various studies in the literature. The items in Table 1 represent the measures that senior managers/directors and chief executives/leaders in an organization must take prior to Six Sigma implementation.

\section{Research Methodology}

This research was an applied, quantitative study based on the framework of analytic survey. The first statistical population of this research included academic experts with knowledge in field of Six Sigma. Twelve of them were selected through purposeful sampling. The second statistical population was composed of experts in the field of the steel industry (Tabarestan Steel Company). The census method 
was used to select a sample because of the low community size (12 individuals). The participants were senior managers, middle managers, and professional employees.

\subsection{Delphi Fuzzy}

In this study, the method proposed by Liu and Chen (2007) was used to implement the fuzzy Delphi analysis process. The process of this method involved the following steps.

Collet experts' opinions. First, the experts were asked to scrutinize the parameters influencing a given decision in terms of importance, using a qualitatively or, if possible, quantitatively scale (Very Important = 9; Important = 7; Relatively Important = 5; Minimally Important =3; Not Important = 1).

Calculate fuzzy numbers. To calculate fuzzy numbers, $\alpha_{\mathrm{ij}}$ values were considered, based on the opinions expressed by the experts (Ataei, 2010). Fuzzy numbers at this stage could be calculated based on different membership functions such as the triangular or trapezoidal state. Considering the high application and simplicity of the triangular method, a fuzzy number was defined through the following relations (Liu and Chen, 2007).

$$
\begin{aligned}
\alpha_{i j} & =(\alpha i j, d i j, g i j) \\
\alpha_{i j} & =\operatorname{Min}\left(\mathrm{b}_{i j k}\right), k=1,2, \ldots . ., n \\
d_{i j} & =\left(\prod_{k=1}^{n} b_{i j k}\right), k=1,2, \ldots . ., n \\
g_{i j} & =\operatorname{Max}\left(b_{i j k}\right), k=1,2, \ldots . ., n
\end{aligned}
$$

where $b_{i j k}$ is the relative importance of parameter $i$ with respect to parameter $j, K$ is experts' points of, $\alpha_{i j}$ and $\mathrm{g}_{i j}$ are the upper and lower limits of the queries, and $d_{i j}$ is the geometric mean of the opinions of the respondents. The components of the fuzzy number were defined according to this format. $\alpha_{\mathrm{ij}}, \mathrm{g}_{\mathrm{ij}}$, $\mathrm{d}_{\mathrm{ij} .}$. The values of these components always fell within this range $[9,1.9]$.

Create a fuzzy inverse matrix. At this stage, according to the fuzzy numbers obtained in the previous step, the fuzzy pair matrix between the various parameters was described through the following relation (Liu and Chen, 2007). Partial fuzzy weight calculation parameters were used to calculate the relative weights of the parameters of the following relations.

$$
\begin{aligned}
A_{i j} & =\left[a_{i j}\right], a_{i j} \times a_{j i} \approx 1, \forall_{i, j}=1,2,3, \ldots . . \\
Z_{i} & =\left[a_{i j} \otimes \ldots \ldots \ldots \otimes a_{i j}\right]^{-1} \\
W_{i} & =Z_{i}\left(Z_{i} \oplus \ldots \ldots \ldots \oplus Z_{n}\right) \\
W_{i} & =\left(\prod_{k=1}^{3 n} W_{i j}\right)
\end{aligned}
$$

where $\otimes$ is the symbol of the multiplication of fuzzy numbers, and $\bigoplus$ is the symbol of the sum of fuzzy numbers, and is a vector of a row representing the fuzzy weight of parameter $i$.

Calculate non-fuzzy weight parameters. In this step, in order to defuzzify the parameters, the geometric mean of the components of the fuzzy weights of the parameters was obtained; as such, the weights of the parameters were formulated as definite numbers (see Liu and Chen, 2007).

\subsection{DEMATEL fuzzy method}

The DEMATEL technique is a method for complex problems first introduced by American scientists between 1926 and 1972; this technique was built based on the theory of graph that was able to solve problems with a simple method. However, there was a problem with the DEMATEL technique, as it relied on a fuzzy DEMATEL technique, which made decisions in conditions of uncertainty. Fuzzy 
DEMATEL makes it easy to make decisions in terms of environmental uncertainty through language variables. This technique can be easily applied to such fields as production, organization management, information systems, and environmental uncertainty (Zhou \& et al. 2011). Furthermore, this technique can solve all problems that organizations confront in group decision-making under fuzzy conditions (Reyes et al., 2011). This method involves the following steps.

Create a matrix of direct relationships between system factors. Experts, using the linguistic variables, expressed their views about the mutual impact of the main factors on each other. By converting linguistic estimates into fuzzy numbers, the primary direct relation matrix $A=\left[a_{i j}\right]$ was created, in which $\mathrm{A}$ is the nonnegative matrix $\mathrm{N} \times \mathrm{N}$, and the frame $a_{i j}$ is a triangular fuzzy number representing the direct impact of factor $i$ on factor $j$. When $i=j$, the diameter components of the matrix are zero (Cheng et al., 2009),

$$
A=\left[\begin{array}{lll}
a_{11} & a_{1 j} & a_{1 n} \\
a_{i 1} & a_{i j} & a_{i n} \\
a_{n 1} & a_{n j} & a_{n n}
\end{array}\right]
$$

Gather the data and merge respondents' opinions. In this step, to determine the effect of each criterion on another, the symbol $0_{\mathrm{ij}}=\left(1_{\mathrm{ij}}, \mathrm{m}_{\mathrm{ij}}, \mathrm{u}_{\mathrm{ij}}\right)$ was used to represent the respondents' comments on the effect of factor $i$ on factor $j$. Where $i=j$ in the matrix, the number was zero. For every respondent, a matrix $n^{*} n b$, a with fuzzy frame, was defined as $O^{\sim p}=\left[O^{\sim p}{ }_{i j}\right]$, where $p$ is the number of respondents and $n$ is the number of factors under investigation. As a result, $O^{\sim 1}, O^{\sim 2}, O^{\sim 3}, \ldots, O^{\sim p}$ were the matrices of responder $p$.

Make the initial decision matrix (O). From the simple average, the comments of all individuals with triangular fuzzy dimensions were $\mathrm{O}_{\mathrm{ij}}=\left(\mathrm{l}_{\mathrm{ij}}, \mathrm{m}_{\mathrm{ij}}\right.$, $\left.\mathrm{u}_{\mathrm{ij}}\right)$ (Jassbi et al., 2010).

$$
\begin{gathered}
\tilde{O}_{i j}=\frac{1}{p} \sum_{p=1}^{p} \tilde{a}_{i j}^{p} \\
\tilde{O}=\left[\begin{array}{cccccc}
\tilde{o}_{11} & \tilde{o}_{12} & \tilde{o}_{13} & \cdot & \cdot & \tilde{o}_{1 n} \\
\tilde{o}_{21} & \tilde{o}_{22} & \tilde{o}_{23} & \cdot & \cdot & \tilde{o}_{2 n} \\
\tilde{o}_{31} & \tilde{o}_{32} & \tilde{o}_{33} & \cdot & \cdot & \tilde{o}_{3 n} \\
\cdot & \cdot & \cdot & \cdot & \cdot & \cdot \\
\cdot & \cdot & \cdot & \cdot & \cdot & \cdot \\
\tilde{o}_{m 1} & \tilde{o}_{m 2} & \tilde{o}_{m 3} & \cdot & \cdot & \tilde{o}_{m n}
\end{array}\right]
\end{gathered}
$$

Calculate the normalized matrix. To create the normalized matrix, the following relations were utilized (Zhou et al., 2011).

$$
\begin{aligned}
Z_{h}^{\sim} & =k \times O_{h}^{\sim} \\
k & =\min \left[\frac{1}{\operatorname{Max} \sum_{j=1}^{n}\left|\tilde{o}_{i j}\right|}, \frac{1}{\operatorname{Max} \sum_{i=1}^{n}\left|\tilde{o}_{i j}\right|}\right] \\
i, j \in\{1,2,3, \ldots \ldots, n\} &
\end{aligned}
$$




$$
\tilde{z}=\left[\begin{array}{cccccc}
\tilde{z}_{11} & \tilde{z}_{12} & \tilde{z}_{13} & \cdot & \cdot & \tilde{z}_{1 n} \\
\tilde{z_{21}} & \tilde{z}_{22} & \tilde{z_{23}} & \cdot & \cdot & \tilde{z}_{2 n} \\
\tilde{z_{31}} & \tilde{z_{32}} & \tilde{z_{33}} & \cdot & \cdot & \tilde{z}_{3 n} \\
\cdot & \cdot & \cdot & \cdot & \cdot & \cdot \\
\cdot & \cdot & \cdot & \cdot & \cdot & \cdot \\
\tilde{z_{m 1}} & \tilde{z}_{m 2} & \tilde{z_{m 3}} & \cdot & \cdot & \tilde{z}_{m n}
\end{array}\right]
$$

Create matrix V. Matrix V was calculated for every fuzzy limit $\left(\mathrm{l}_{\mathrm{ij}}, \mathrm{m}_{\mathrm{ij}} \mathrm{j}, \mathrm{u}\right.$ "ij $)$ via.

$$
\begin{aligned}
& l_{i j}^{\prime \prime}=\tilde{z_{l}} \times\left(I-\tilde{z_{l}}\right)^{-1} \\
& m_{i j}^{\prime \prime}=\tilde{z_{m}} \times\left(I-\tilde{z_{m}}\right)^{-1} \\
& u_{i j}^{\prime \prime}=\tilde{z_{u}} \times\left(I-\tilde{z_{u}}\right)^{-1}
\end{aligned}
$$

Finally, each of the lower, middle, and upper triangles were bound together, as a result of which matrix $V^{\sim}$ was formulated.

$$
\tilde{V}=\left[\begin{array}{cccccc}
\tilde{v_{11}} & \tilde{v_{12}} & \tilde{v_{13}} & \cdot & \cdot & \tilde{v_{1 n}} \\
\tilde{v_{21}} & \tilde{v_{22}} & \tilde{v_{23}} & \cdot & \cdot & \tilde{v}_{2 n} \\
\tilde{v_{31}} & \tilde{v_{32}} & \tilde{v_{33}} & \cdot & \cdot & \tilde{v_{3 n}} \\
\cdot & \cdot & \cdot & \cdot & \cdot & \cdot \\
\cdot & \cdot & \cdot & \cdot & \cdot & \cdot \\
\tilde{v_{m 1}} & \tilde{v_{m 2}} & \tilde{v_{m 3}} & \cdot & \cdot & \tilde{v}_{m n}
\end{array}\right]
$$

Fuzzify non-fuzzy numbers. For this purpose, $i$ and $j$ were used, according to the following relations.

$$
v=\frac{(l+4 m+u)}{6}
$$

As a result of which we have.

$$
V=\left[\begin{array}{cccccc}
v_{11} & v_{12} & v_{13} & \cdot & \cdot & v_{1 n} \\
v_{21} & v_{22} & v_{23} & . & \cdot & v_{2 n} \\
v_{31} & v_{32} & v_{33} & \cdot & \cdot & v_{3 n} \\
\cdot & \cdot & \cdot & \cdot & \cdot & \cdot \\
\cdot & \cdot & \cdot & \cdot & \cdot & \cdot \\
v_{m 1} & v_{m 2} & v_{m 3} & \cdot & \cdot & v_{m n}
\end{array}\right]
$$

\subsection{Fuzzy ANP method}

The ANP Analytic network process proposed by Saaty (2004) deals with multi-criteria decisionmaking problems in which there is an interaction and interdependence among decision-making criteria (or opinions, sub-criteria). In the ANP technique, to demonstrate the existing dependencies of decisionmaking levels, a supermatrix is normally constructed. In this study, a grid and a supermatrix were used (see Fig. 1). 


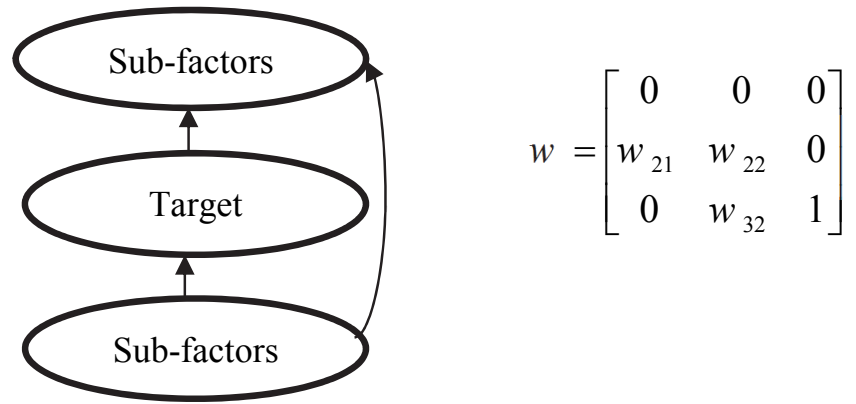

Fig 1. The grid used in this research and its supermatrix

The first level of the network included the goals, and the second level addressed the main factors which had Zornian affiliations and were placed at the third level. In the supermatrix $\mathrm{W}, W 21$ was the relative weight of the main factors with respect to the target node $W 22$. The internal weight between the main W32 factors was the weight of the subframes relative to their respective principal factors. The matrix $\mathrm{T}$ was the output of the DEMATEL method; after normalization, it was considered to be the matrix W22. To calculate $W 21$ and $W 3$, pairwise comparisons were made. To calculate fuzzy numbers, $\alpha_{\mathrm{ij}}$ values were considered. Expert opinions were processed directly (Ataei, 2010). Fuzzy numbers at this stage could be calculated based on different membership functions such as triangular or trapezoidal formats. Given the high application and simplicity of working with the triangular method, a fuzzy number was defined through the following relations (Liu \& Chen, 2007).

$$
\begin{aligned}
\alpha_{i j} & =(\alpha i j, d i j, g i j) \\
\alpha_{i j} & =\operatorname{Min}\left(\mathrm{b}_{i j k}\right), k=1,2, \ldots ., n \\
d_{i j} & =\left(\prod_{k=1}^{n} b_{i j k}\right), k=1,2, \ldots . ., n \\
g_{i j} & =\operatorname{Max}\left(b_{i j k}\right), k=1,2, \ldots . ., n
\end{aligned}
$$

In the development analysis method, for each row of the pairwise comparison matrix, the $S_{k}$ value, which was itself a triangular number, was calculated via.

$$
S_{k}=\sum_{j=1}^{n} M_{k j} *\left[\sum_{i=1}^{m} \sum_{j=1}^{n} M_{i j}\right]^{-1},
$$

where $k$ is the row number, while $i$ and $j$ indicate the options and indicators, respectively. In general, in the development analysis method, after the calculation of $S_{k}$, have to be obtained, the magnitude of items with respect to each other must be computed. If $\mathrm{M}_{1}$ and $\mathrm{M}_{2}$ are two triangular fuzzy numbers, the magnitude of $\mathrm{M}_{1}$ over $\mathrm{M}_{2}$, shown by $V\left(M_{1} \geq M_{2}\right)$, is decided as follows.

$$
\left\{\begin{array}{l}
V\left(m_{1} \geq m_{2}\right)=1 \\
\text { if } m_{1} \geq m_{2} \\
V\left(m_{1} \geq m_{2}=\operatorname{hgt}\left(m_{1} \cap m_{2}\right)\right. \\
\text { otherwise }
\end{array}\right\}
$$

we have,

$$
\operatorname{hgt}\left(M_{1} \cap M_{2}\right)=\frac{u_{1}-l_{2}}{\left(u_{1}-l_{2}\right)+\left(m_{2}-m_{1}\right)}
$$


The formula could also be computed through the equation below. The size of a triangular fuzzy number which is larger than of another triangular fuzzy number $k$ is verified through Eq. (28).

$$
V\left(M_{1} \geq M_{2}, \ldots, M_{K}\right)=V\left(M_{1} \geq M_{2}\right), \ldots, V\left(M_{1} \geq M_{K}\right)
$$

To make the weights of the indicators in the pairwise comparison matrix, relation 28 was used.

$$
W^{\prime}\left(x_{i}\right)=\operatorname{Min}\left\{V\left(S_{i} \geq S_{k}\right)\right\}, \quad k=1,2, \ldots, n . \quad k \neq i
$$

The weight vector of the indices was then formulated as follows,

$$
W^{\prime}\left(x_{i}\right)=\left[W^{\prime}\left(c_{1}\right), W^{\prime}\left(c_{2}\right), \ldots, W^{\prime}\left(c_{n}\right)\right]^{T},
$$

which is the vector of abnormal coefficients of the fuzzy analytic hierarchical process. Based on the following equation, the abnormal results obtained from the relation above are normalized. The normalized results were obtained through the following equation.

$$
W_{i}=\frac{w_{i}^{\prime}}{\sum w_{i}^{\prime}}
$$

where Factors is the vector of the abnormal coefficients of the fuzzy hierarchy process. Through the following relation, the results were obtained with a view to the above normal relation. The normalized results were calculated via.

$$
w_{c}=B W
$$

The complete solution to the network analysis process, and even the hierarchical analysis process, can only be used in a real and precise method when the number of criteria and options are limited.

\section{Findings}

The findings of the statistical and analytic procedures are divided into three parts, which are reported in the following sub-sections.

\subsection{Part 1. Delphi Fuzzy Findings}

In this sub-section, the indicators extracted from the literature were processed through the fuzzy Delphi, which involved four stages. In the first stage, according to the options shared, the difference between the two stages was less than the threshold value $(0.1)$, in which case, the polling process was terminated (Cheng \& Lin, 2002). The indicators were used for finalization and the final indicators were also found (see Table 1). 
Table 1

Indicators extracted from the literature $\&$ their initial and finalized list

\begin{tabular}{|c|c|c|c|c|c|c|c|c|c|c|c|c|c|c|c|c|c|c|c|}
\hline $\begin{array}{l}\text { Readiness } \\
\text { variables }\end{array}$ & 1 & 2 & 3 & 4 & 5 & 6 & 7 & 8 & 9 & 10 & 11 & 12 & $\begin{array}{r}\text { first } \\
\text { fuzzy } \\
\text { number } \\
(\mathrm{MIN})\end{array}$ & $\begin{array}{r}\text { second fuzzy } \\
\text { number } \\
\text { (GEOMEAN) }\end{array}$ & $\begin{array}{r}\text { third } \\
\text { fuzzy } \\
\text { number } \\
(\mathrm{MAX})\end{array}$ & $\begin{array}{l}\text { defuzzied } \\
{ }^{1} \text { Number }\end{array}$ & 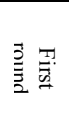 & 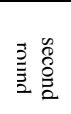 & 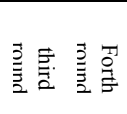 \\
\hline 1. & 3 & 4 & 4 & 4 & 4 & 3 & 4 & 4 & 4 & 3 & 4 & 3 & 3 & 3.634241 & 4 & 2.90856 & $\sqrt{ }$ & & \\
\hline 2. & 4 & 3 & 3 & 2 & 3 & 3 & 3 & 3 & 3 & 4 & 3 & 3 & 2 & 3.042777 & 4 & 1.760694 & $\sqrt{ }$ & & \\
\hline 3. & 4 & 4 & 3 & 3 & 4 & 4 & 4 & 3 & 3 & 4 & 4 & 3 & 3 & 3.548152 & 4 & 2.887038 & $\sqrt{ }$ & & \\
\hline 4. & 3 & 4 & 4 & 4 & 4 & 4 & 4 & 3 & 3 & 3 & 4 & 4 & 3 & 3.634241 & 4 & 2.90856 & $\sqrt{ }$ & & \\
\hline 5. & 3 & 4 & 4 & 5 & 3 & 3 & 4 & 4 & 4 & 4 & 4 & 3 & 3 & 3.702453 & 5 & 2.675613 & $\sqrt{ }$ & & \\
\hline 6. & 4 & 3 & 3 & 3 & 3 & 4 & 3 & 4 & 4 & 3 & 3 & 4 & 3 & 3.382043 & 4 & 2.845511 & & & \\
\hline 7. & 4 & 3 & 4 & 3 & 3 & 3 & 4 & 3 & 4 & 4 & 3 & 4 & 3 & 3.464102 & 4 & 2.866025 & & & \\
\hline 8. & 3 & 4 & 3 & 4 & 4 & 5 & 3 & 4 & 4 & 4 & 4 & 4 & 3 & 3.792287 & 5 & 2.698072 & & & \\
\hline 9. & 3 & 4 & 4 & 4 & 4 & 3 & 3 & 4 & 3 & 4 & 4 & 3 & 3 & 3.548152 & 4 & 2.887038 & $\sqrt{ }$ & & \\
\hline 10. & 4 & 4 & 3 & 4 & 3 & 5 & 5 & 4 & 3 & 3 & 4 & 3 & 3 & 3.682594 & 5 & 2.670649 & & & \\
\hline 11. & 3 & 3 & 3 & 3 & 4 & 4 & 3 & 3 & 3 & 3 & 3 & 4 & 3 & 3.22371 & 4 & 2.805927 & & & \\
\hline 12. & 3 & 3 & 4 & 3 & 3 & 3 & 4 & 4 & 3 & 4 & 3 & 4 & 3 & 3.382043 & 4 & 2.845511 & & & \\
\hline 13. & 4 & 4 & 4 & 3 & 4 & 4 & 4 & 4 & 5 & 4 & 3 & 4 & 3 & 3.884299 & 5 & 2.721075 & $\sqrt{ }$ & & \\
\hline 14. & 4 & 3 & 3 & 4 & 3 & 2 & 3 & 3 & 3 & 4 & 4 & 3 & 2 & 3.192223 & 4 & 1.798056 & & & \\
\hline 15. & 4 & 4 & 3 & 3 & 4 & 5 & 4 & 4 & 5 & 3 & 3 & 3 & 3 & 3.682594 & 5 & 2.670649 & $\sqrt{ }$ & & \\
\hline 16. & 3 & 3 & 3 & 4 & 4 & 3 & 2 & 4 & 3 & 4 & 3 & 3 & 2 & 3.192223 & 4 & 1.798056 & $\sqrt{ }$ & & \\
\hline 17. & 3 & 4 & 4 & 4 & 3 & 4 & 3 & 3 & 4 & 4 & 4 & 4 & 3 & 3.634241 & 4 & 2.90856 & $\sqrt{ }$ & & \\
\hline 18. & 4 & 4 & 4 & 4 & 3 & 3 & 4 & 3 & 3 & 3 & 4 & 3 & 3 & 3.464102 & 4 & 2.866025 & & & \\
\hline 19. & 4 & 4 & 3 & 3 & 4 & 4 & 5 & 4 & 5 & 3 & 4 & 3 & 3 & 3.771946 & 5 & 2.692986 & $\sqrt{ }$ & & \\
\hline 20. & 3 & 3 & 3 & 3 & 4 & 3 & 3 & 4 & 3 & 4 & 3 & 4 & 3 & 3.301927 & 4 & 2.825482 & & & \\
\hline 21. & 3 & 3 & 3 & 4 & 3 & 4 & 4 & 4 & 4 & 3 & 3 & 4 & 3 & 3.464102 & 4 & 2.866025 & & & \\
\hline 22. & 4 & 4 & 4 & 4 & 4 & 5 & 4 & 3 & 3 & 3 & 4 & 4 & 3 & 3.792287 & 5 & 2.698072 & $\sqrt{ }$ & & \\
\hline 23. & 3 & 4 & 3 & 4 & 3 & 3 & 4 & 3 & 4 & 3 & 3 & 3 & 3 & 3.301927 & 4 & 2.825482 & & & \\
\hline 24. & 4 & 3 & 3 & 3 & 4 & 4 & 4 & 4 & 3 & 4 & 4 & 3 & 3 & 3.548152 & 4 & 2.887038 & $\sqrt{ }$ & & \\
\hline 25. & 3 & 3 & 4 & 3 & 3 & 3 & 3 & 3 & 4 & 4 & 4 & 4 & 3 & 3.382043 & 4 & 2.845511 & & & \\
\hline 26. & 3 & 4 & 3 & 3 & 3 & 3 & 3 & 4 & 3 & 3 & 4 & 3 & 3 & 3.22371 & 4 & 2.805927 & & & \\
\hline 27. & 4 & 4 & 3 & 4 & 4 & 3 & 3 & 3 & 3 & 4 & 3 & 4 & 3 & 3.464102 & 4 & 2.866025 & & & \\
\hline 28. & 3 & 4 & 4 & 3 & 4 & 4 & 4 & 4 & 3 & 4 & 4 & 4 & 3 & 3.722419 & 4 & 2.930605 & $\sqrt{ }$ & & \\
\hline 29. & 3 & 3 & 3 & 3 & 3 & 4 & 3 & 4 & 4 & 4 & 3 & 4 & 3 & 3.382043 & 4 & 2.845511 & $\sqrt{ }$ & & \\
\hline 30. & 3 & 3 & 4 & 4 & 3 & 3 & 4 & 5 & 4 & 3 & 3 & 3 & 3 & 3.445521 & 5 & 2.61138 & & & \\
\hline 31. & 4 & 3 & 3 & 4 & 3 & 4 & 4 & 3 & 3 & 4 & 4 & 4 & 3 & 3.548152 & 4 & 2.887038 & $\sqrt{ }$ & & \\
\hline 32. & 4 & 4 & 4 & 4 & 4 & 4 & 4 & 4 & 4 & 4 & 4 & 3 & 3 & 3.905246 & 4 & 2.976312 & & & \\
\hline 33. & 4 & 3 & 4 & 3 & 4 & 3 & 3 & 4 & 4 & 3 & 4 & 3 & 3 & 3.464102 & 4 & 2.866025 & $\sqrt{ }$ & & \\
\hline 34. & 3 & 4 & 4 & 3 & 3 & 4 & 3 & 4 & 4 & 4 & 3 & 4 & 3 & 3.548152 & 4 & 2.887038 & $\sqrt{ }$ & & \\
\hline 35. & 4 & 4 & 3 & 4 & 3 & 4 & 4 & 3 & 3 & 4 & 3 & 4 & 3 & 3.548152 & 4 & 2.887038 & $\sqrt{ }$ & & \\
\hline 36. & 3 & 4 & 3 & 3 & 3 & 3 & 4 & 3 & 4 & 3 & 4 & 4 & 3 & 3.382043 & 4 & 2.845511 & & & \\
\hline 37. & 4 & 3 & 4 & 4 & 4 & 4 & 4 & 3 & 4 & 3 & 4 & 3 & 3 & 3.634241 & 4 & 2.90856 & $\sqrt{ }$ & & \\
\hline 38. & 3 & 3 & 3 & 4 & 3 & 4 & 3 & 4 & 3 & 4 & 4 & 3 & 3 & 3.382043 & 4 & 2.845511 & $\sqrt{ }$ & & \\
\hline 39. & 4 & 4 & 3 & 4 & 3 & 4 & 3 & 4 & 4 & 4 & 3 & 4 & 3 & 3.634241 & 4 & 2.90856 & $\sqrt{ }$ & & \\
\hline 40. & 3 & 4 & 4 & 3 & 4 & 4 & 3 & 3 & 4 & 4 & 3 & 4 & 3 & 3.548152 & 4 & 2.887038 & $\sqrt{ }$ & & \\
\hline 41. & 4 & 4 & 4 & 4 & 3 & 3 & 4 & 3 & 3 & 3 & 4 & 4 & 3 & 3.548152 & 4 & 2.887038 & $\sqrt{ }$ & & \\
\hline 42. & 3 & 3 & 4 & 3 & 3 & 4 & 3 & 4 & 3 & 3 & 3 & 3 & 3 & 3.22371 & 4 & 2.805927 & & & \\
\hline 43. & 4 & 3 & 3 & 3 & 4 & 3 & 3 & 4 & 4 & 4 & 4 & 3 & 3 & 3.464102 & 4 & 2.866025 & $\sqrt{ }$ & & \\
\hline 44. & 3 & 3 & 4 & 4 & 3 & 4 & 4 & 3 & 4 & 3 & 3 & 4 & 3 & 3.464102 & 4 & 2.866025 & & & \\
\hline 45. & 3 & 4 & 4 & 4 & 4 & 3 & 4 & 3 & 4 & 4 & 4 & 3 & 3 & 3.634241 & 4 & 2.90856 & & & \\
\hline 46. & 3 & 3 & 3 & 4 & 3 & 4 & 4 & 4 & 3 & 3 & 4 & 4 & 3 & 3.464102 & 4 & 2.866025 & & & \\
\hline 47. & 4 & 3 & 3 & 3 & 4 & 3 & 3 & 3 & 3 & 4 & 3 & 3 & 3 & 3.22371 & 4 & 2.805927 & & & \\
\hline 48. & 4 & 4 & 4 & 3 & 4 & 4 & 3 & 4 & 4 & 4 & 3 & 4 & 3 & 3.722419 & 4 & 2.930605 & & & \\
\hline 49. & 4 & 4 & 4 & 4 & 4 & 3 & 4 & 3 & 3 & 5 & 4 & 4 & 3 & 3.792287 & 5 & 2.698072 & & & \\
\hline 50. & 3 & 4 & 3 & 4 & 3 & 4 & 3 & 4 & 4 & 3 & 4 & 3 & 3 & 3.464102 & 4 & 2.866025 & & & \\
\hline 51. & 4 & 3 & 4 & 4 & 3 & 4 & 4 & 3 & 3 & 4 & 4 & 3 & 3 & 3.548152 & 4 & 2.887038 & & & \\
\hline 52. & 3 & 3 & 3 & 3 & 4 & 4 & 4 & 4 & 4 & 4 & 3 & 4 & 3 & 3.548152 & 4 & 2.887038 & & & \\
\hline 53. & 4 & 4 & 4 & 3 & 3 & 3 & 4 & 4 & 4 & 4 & 3 & 4 & 3 & 3.634241 & 4 & 2.90856 & $\sqrt{ }$ & & \\
\hline 54. & 3 & 3 & 3 & 4 & 3 & 4 & 3 & 3 & 5 & 3 & 3 & 4 & 3 & 3.363902 & 5 & 2.590975 & $\sqrt{ }$ & & \\
\hline 55. & 3 & 4 & 4 & 3 & 4 & 4 & 4 & 4 & 3 & 3 & 4 & 3 & 3 & 3.548152 & 4 & 2.887038 & & & \\
\hline 56. & 4 & 4 & 3 & 4 & 4 & 3 & 3 & 3 & 4 & 3 & 4 & 3 & 3 & 3.464102 & 4 & 2.866025 & & & \\
\hline 57. & 3 & 4 & 4 & 3 & 4 & 4 & 3 & 4 & 4 & 4 & 3 & 3 & 3 & 3.548152 & 4 & 2.887038 & & & \\
\hline 58. & 4 & 3 & 3 & 4 & 3 & 4 & 4 & 4 & 4 & 4 & 4 & 4 & 3 & 3.722419 & 4 & 2.930605 & $\sqrt{ }$ & & \\
\hline 59. & 3 & 4 & 3 & 4 & 4 & 3 & 4 & 4 & 3 & 3 & 4 & 4 & 3 & 3.548152 & 4 & 2.887038 & $\sqrt{ }$ & & \\
\hline 60. & 3 & 3 & 4 & 3 & 4 & 3 & 4 & 3 & 3 & 3 & 3 & 3 & 3 & 3.22371 & 4 & 2.805927 & $\sqrt{ }$ & & \\
\hline 61. & 4 & 3 & 3 & 3 & 3 & 4 & 3 & 3 & 3 & 4 & 4 & 4 & 3 & 3.382043 & 4 & 2.845511 & $\sqrt{ }$ & & \\
\hline 62. & 4 & 4 & 4 & 4 & 3 & 3 & 3 & 4 & 4 & 4 & 4 & 3 & 3 & 3.634241 & 4 & 2.90856 & & & \\
\hline 63. & 4 & 4 & 3 & 4 & 4 & 4 & 4 & 4 & 4 & 3 & 5 & 3 & 3 & 3.792287 & 5 & 2.698072 & & $\sqrt{ }$ & \\
\hline 64. & 3 & 4 & 4 & 4 & 4 & 3 & 4 & 3 & 3 & 3 & 3 & 4 & 3 & 3.464102 & 4 & 2.866025 & & $\sqrt{ }$ & \\
\hline 65. & 3 & 3 & 3 & 3 & 3 & 3 & 4 & 3 & 3 & 4 & 4 & 4 & 3 & 3.301927 & 4 & 2.825482 & & $\sqrt{ }$ & \\
\hline 66. & 3 & 3 & 4 & 3 & 4 & 4 & 3 & 4 & 4 & 3 & 4 & 3 & 3 & 3.464102 & 4 & 2.866025 & & & \\
\hline 67. & 4 & 4 & 3 & 3 & 3 & 4 & 3 & 3 & 4 & 4 & 4 & 4 & 3 & 3.548152 & 4 & 2.887038 & & & \\
\hline 68. & 4 & 4 & 3 & 4 & 4 & 3 & 4 & 4 & 3 & 3 & 3 & 3 & 3 & 3.464102 & 4 & 2.866025 & & $\sqrt{ }$ & \\
\hline 69. & 3 & 4 & 4 & 4 & 3 & 3 & 4 & 3 & 3 & 4 & 3 & 5 & 3 & 3.52912 & 5 & 2.63228 & & $\sqrt{ }$ & \\
\hline 70. & 3 & 3 & 3 & 3 & 4 & 4 & 3 & 4 & 4 & 3 & 3 & 3 & 3 & 3.301927 & 4 & 2.825482 & & $\sqrt{ }$ & \\
\hline 71. & 3 & 3 & 3 & 4 & 3 & 4 & 3 & 3 & 3 & 4 & 4 & 5 & 3 & 3.445521 & 5 & 2.61138 & & & \\
\hline 72. & 4 & 4 & 4 & 3 & 4 & 3 & 3 & 4 & 4 & 4 & 4 & 4 & 3 & 3.722419 & 4 & 2.930605 & & & \\
\hline 73. & 4 & 3 & 4 & 3 & 3 & 4 & 4 & 4 & 3 & 3 & 3 & 3 & 3 & 3.382043 & 4 & 2.845511 & & & \\
\hline 74. & 3 & 4 & 4 & 4 & 4 & 4 & 4 & 3 & 4 & 4 & 3 & 4 & 3 & 3.722419 & 4 & 2.930605 & & $\sqrt{ }$ & \\
\hline 75. & 3 & 4 & 4 & 4 & 5 & 4 & 4 & 4 & 4 & 3 & 4 & 4 & 3 & 3.884299 & 5 & 2.721075 & & $\sqrt{ }$ & \\
\hline
\end{tabular}

${ }^{1} \mathrm{Min}+(($ GEOMEAN+MAX)/4 $)$ 
The final indicators are summarized in Table 2 as follows,

Table 2

Final indicators

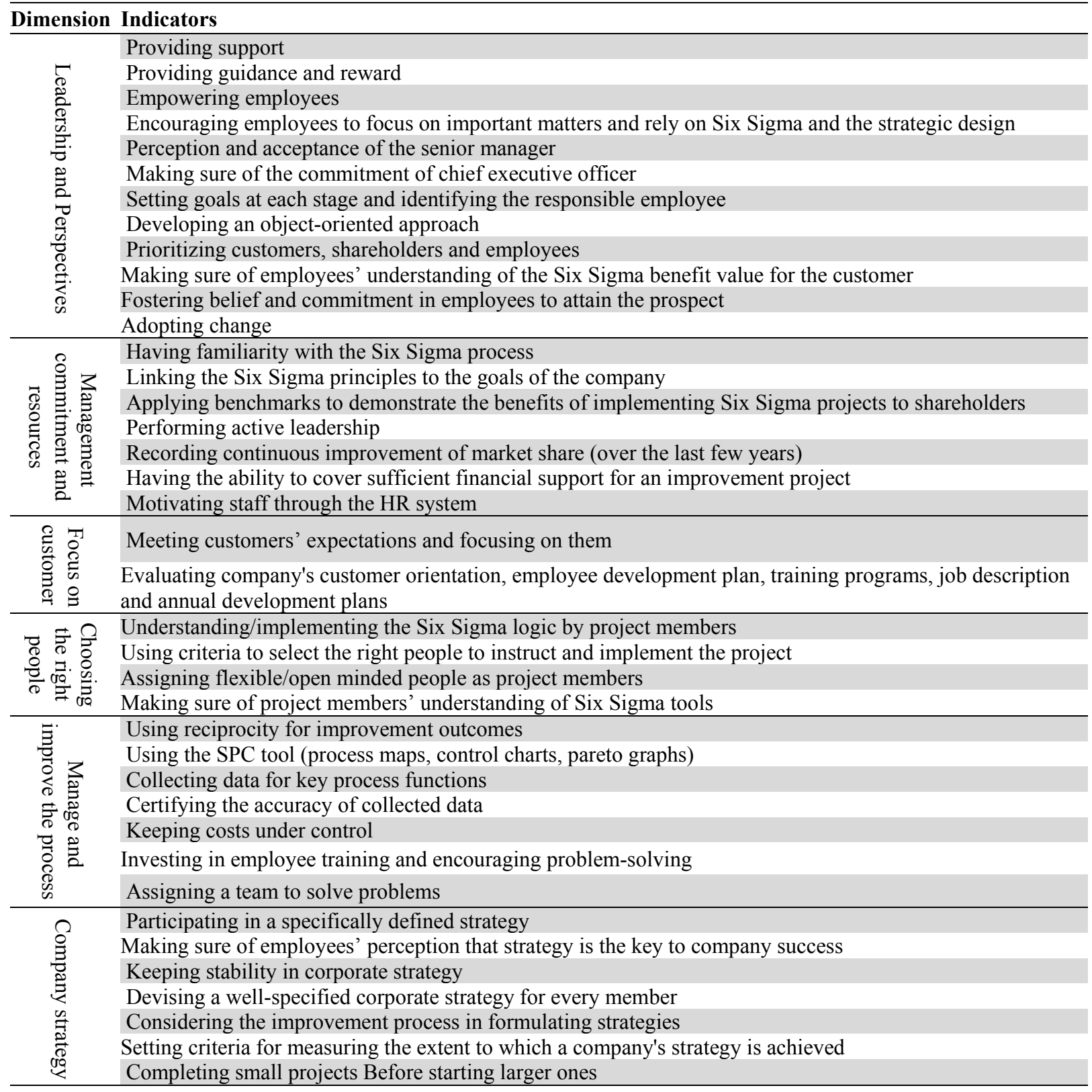

The final indicators presented in Table 2 indicate that the Tabarestan Steel Co. had to use these indicators to be able to adopt and implement Six Sigma projects.

\subsection{DEMATEL fuzzy findings}

This section is presented step-by-step according to the explanations provided. Due to constraint of space, only one sample analysis is presented. The rest of the information is reported in tables (see table 3 ). 
Table 3

List of the criteria

\begin{tabular}{lll}
\hline Row & Symbol & Criteria \\
\hline 1 & $\mathrm{C}_{1}$ & Leadership and Perspectives \\
2 & $\mathrm{C}_{2}$ & Management commitment and resources \\
3 & $\mathrm{C}_{3}$ & Focus on customer \\
4 & $\mathrm{C}_{4}$ & Choosing the right people \\
5 & $\mathrm{C}_{5}$ & Manage and improve the process \\
6 & $\mathrm{C}_{6}$ & company strategy \\
\hline
\end{tabular}

To verify the criteria used in the 12 expert opinions, Table 4 shows the paired comparison of each expert. In this matrix, $\tilde{x}_{i j}=\left(l_{i j}, m_{i j}, u_{i j}\right)$ are fuzzy triangular numbers, and $\tilde{x}_{i i}=(i=1,2,3, \ldots, n)$ are considered to be fuzzy numbers $(0,0,0)$. Accordingly, expert opinions were collected and combined through the relations mentioned in methodology section.

\section{Table 4}

Average of expert opinions

\begin{tabular}{lcccccc}
\hline \multicolumn{2}{c}{$\mathrm{C}_{1}$} & $\mathrm{C}_{2}$ & $\mathrm{C}_{3}$ & $\mathrm{C}_{4}$ & $\mathrm{C}_{5}$ \\
\hline $\mathrm{C}_{1}$ & $(0.0000,0.0000,0.0000)$ & $(3.0000,3.7224,4.0000)$ & $(3.0000,3.7224,4.0000)$ & $(2.0000,3.1166,4.0000)$ & $(2.0000,3.4303,4.0000)$ & $(3.0000,3.4641,4.0000)$ \\
$\mathrm{C}_{2}$ & $(2.0000,2.2134,3.0000)$ & $(0.0000,0.0000,0.0000)$ & $(3.0000,3.4641,4.0000)$ & $(2.0000,2.8563,4.0000)$ & $(3.0000,3.2237,4.0000)$ & $(3.0000,3.5482,4.0000)$ \\
$\mathrm{C}_{3}$ & $(3.0000,3.0728,4.0000)$ & $(3.0000,3.0728,4.0000)$ & $(0.0000,0.0000,0.0000)$ & $(2.0000,2.9417,4.0000)$ & $(2.0000,3.1302,4.0000)$ & $(2.0000,3.2697,4.0000)$ \\
$\mathrm{C}_{4}$ & $(2.0000,2.0687,3.0000)$ & $(2.0000,2.0687,3.0000)$ & $(3.0000,3.1473,4.0000)$ & $(0.0000,0.0000,0.0000)$ & $(2.0000,2.0687,3.0000)$ & $(3.0000,3.5482,4.0000)$ \\
$\mathrm{C}_{5}$ & $(3.0000,3.0728,4.0000)$ & $(3.0000,3.0728,4.0000)$ & $(3.0000,3.0728,4.0000)$ & $(2.0000,2.0687,3.0000)$ & $(0.0000,0.0000,0.0000)$ & $(3.0000,3.3019,4.0000)$ \\
$\mathrm{C}_{6}$ & $(3.0000,3.0728,4.0000)$ & $(3.0000,3.1473,4.0000)$ & $(4.0000,4.0000,4.0000)$ & $(3.0000,3.0728,4.0000)$ & $(3.0000,3.0728,4.0000)$ & $(0.0000,0.0000,0.0000)$ \\
\hline
\end{tabular}

Based on the relations mentioned, the views were integrated in the normalization section in the fuzzy metamaterial and matrix. Table 5 shows the normalized matrix.

Table 5

Normalized Matrix

\begin{tabular}{ccccccc}
\hline & $\mathrm{C} 1$ & $\mathrm{C} 2$ & $\mathrm{C} 3$ & $\mathrm{C} 4$ & $\mathrm{C} 5$ & \\
\hline C1 & $(0.0000,0.0000,0.0000)$ & $(0.1500,0.1861,0.2000)$ & $(0.1500,0.1861,0.2000)$ & $(0.1000,0.1558,0.2000)$ & $(0.1000,0.1715,0.2000)$ & $(0.1500,0.1732,0.2000)$ \\
C2 & $(0.1000,0.1107,0.1500)$ & $(0.0000,0.0000,0.0000)$ & $(0.1500,0.1732,0.2000)$ & $(0.1000,0.1428,0.2000)$ & $(0.1500,0.1612,0.2000)$ & $(0.1500,0.1774,0.2000)$ \\
C3 & $(0.1500,0.1536,0.2000)$ & $(0.1500,0.1536,0.2000)$ & $(0.0000,0.0000,0.0000)$ & $(0.1000,0.1471,0.2000)$ & $(0.1000,0.1565,0.2000)$ & $(0.1000,0.1635,0.2000)$ \\
C4 & $(0.1000,0.1034,0.1500)$ & $(0.1000,0.1034,0.1500)$ & $(0.1500,0.1574,0.2000)$ & $(0.0000,0.0000,0.0000)$ & $(0.1000,0.1034,0.1500)$ & $(0.1500,0.1774,0.2000)$ \\
C5 & $(0.1500,0.1536,0.2000)$ & $(0.1500,0.1536,0.2000)$ & $(0.1500,0.1536,0.2000)$ & $(0.1000,0.1034,0.1500)$ & $(0.0000,0.0000,0.0000)$ & $(0.1500,0.1651,0.2000)$ \\
C6 & $(0.1500,0.1536,0.2000)$ & $(0.1500,0.1574,0.2000)$ & $(0.2000,0.2000,0.2000)$ & $(0.1500,0.1536,0.2000)$ & $(0.1500,0.1536,0.2000)$ & $(0.0000,0.0000,0.0000)$ \\
\hline
\end{tabular}

After calculating the above matrix, the total fuzzy relations were normalized according to the relations mentioned in the fuzzy demultiplex section. Table 6 shows the matrix $\mathrm{V}$.

Table 6

Total relationships matrix

\begin{tabular}{ccccccc}
\hline & $\mathrm{C}_{1}$ & $\mathrm{C}_{2}$ & $\mathrm{C}_{3}$ & $\mathrm{C}_{4}$ & $\mathrm{C}_{5}$ & $\mathrm{C}_{6}$ \\
\hline $\mathrm{C}_{1}$ & $(0.2270,0.4437,3.6667)$ & $(0.3737,0.6438,4.0000)$ & $(0.4021,0.7078,4.1667)$ & $(0.2859,0.5920,4.0000)$ & $(0.3028,0.6292,4.0000)$ & $(0.3686,0.6889,4.1667)$ \\
$\mathrm{C}_{2}$ & $(0.3203,0.4957,3.6389)$ & $(0.2440,0.4318,3.6667)$ & $(0.4029,0.6375,3.9931)$ & $(0.2864,0.5313,3.8333)$ & $(0.3430,0.5677,3.8333)$ & $(0.3693,0.6321,3.9931)$ \\
$\mathrm{C}_{3}$ & $(0.3400,0.5333,3.8333)$ & $(0.3554,0.5716,4.0000)$ & $(0.2503,0.4966,4.0000)$ & $(0.2701,0.5404,4.0000)$ & $(0.2863,0.5703,4.0000)$ & $(0.3128,0.6285,4.1667)$ \\
$\mathrm{C}_{4}$ & $(0.3033,0.4360,3.3333)$ & $(0.3171,0.4675,3.4783)$ & $(0.3838,0.5596,3.6594)$ & $(0.1819,0.3521,3.3478)$ & $(0.2873,0.4646,3.4783)$ & $(0.3518,0.56665,3.6594)$ \\
$\mathrm{C}_{5}$ & $(0.3737,0.5163,3.6944)$ & $(0.3907,0.5534,3.8551)$ & $(0.4204,0.6081,4.0142)$ & $(0.2988,0.4888,3.8188)$ & $(0.2257,0.4168,3.6884)$ & $(0.3854,0.6076,4.0142)$ \\
$\mathrm{C}_{6}$ & $(0.4016,0.5528,3.8333)$ & $(0.4199,0.5954,4.0000)$ & $(0.4915,0.6878,4.1667)$ & $(0.3620,0.5655,4.0000)$ & $(0.3811,0.5891,4.0000)$ & $(0.2838,0.5114,4.0000)$ \\
\hline
\end{tabular}

To be able to apply the relations obtained from the fuzzy DEMATEL to the fuzzy network analysis process, the entire interface matrix was defuzzified. Therefore, the matrix primarily defined the relationships and was finally normalized within a column (see Table 7). Table 8 illustrates the relationships within the frame of the normalized matrix. 
Table 7

Abnormalized diaphragmatic relationships matrix

\begin{tabular}{lcccccc}
\hline & $\mathrm{C}_{1}$ & $\mathrm{C}_{2}$ & $\mathrm{C}_{3}$ & $\mathrm{C}_{4}$ & $\mathrm{C}_{5}$ & $\mathrm{C}_{6}$ \\
\hline $\mathrm{C}_{1}$ & 0.9447 & 1.1582 & 1.2333 & 1.1090 & 1.1366 & 1.2152 \\
$\mathrm{C}_{2}$ & 0.9903 & 0.9397 & 1.1577 & 1.0408 & 1.0745 & 1.1485 \\
$\mathrm{C}_{3}$ & 1.0511 & 1.1070 & 1.0395 & 1.0720 & 1.0946 & 1.1656 \\
$\mathrm{C}_{4}$ & 0.8968 & 0.9442 & 1.0469 & 0.8230 & 0.9373 & 1.0462 \\
$\mathrm{C}_{5}$ & 1.0222 & 1.0766 & 1.1445 & 1.0121 & 0.9302 & 1.1383 \\
$\mathrm{C}_{6}$ & 1.0744 & 1.1336 & 1.2349 & 1.1040 & 1.1229 & 1.0549 \\
\hline
\end{tabular}

Table 8

Normalized diaphragmatic relationships matrix

\begin{tabular}{lcccccc}
\hline & $\mathrm{C}_{1}$ & $\mathrm{C}_{2}$ & $\mathrm{C}_{3}$ & $\mathrm{C}_{4}$ & $\mathrm{C}_{5}$ & $\mathrm{C}_{6}$ \\
\hline $\mathrm{C}_{1}$ & 0.15799 & 0.18213 & 0.17987 & 0.18001 & 0.18052 & 0.17953 \\
$\mathrm{C}_{2}$ & 0.16562 & 0.14777 & 0.16884 & 0.16894 & 0.17066 & 0.16968 \\
$\mathrm{C}_{3}$ & 0.17578 & 0.17408 & 0.15160 & 0.17400 & 0.17385 & 0.17220 \\
$\mathrm{C}_{4}$ & 0.14998 & 0.14848 & 0.15268 & 0.13358 & 0.14887 & 0.15456 \\
$\mathrm{C}_{5}$ & 0.17095 & 0.16930 & 0.16691 & 0.16428 & 0.14774 & 0.16817 \\
$\mathrm{C}_{6}$ & 0.17968 & 0.17826 & 0.18010 & 0.17919 & 0.17835 & 0.15585 \\
\hline
\end{tabular}

\subsection{Fuzzy Analytic Network Process Findings}

\subsubsection{Create a comparative matrix}

This section is divided into seven sub-parts, including weighting and dimensioning information. The subcomponents were analyzed according to the method of fuzzy network analysis process, and were further developed to analyze the identification of the effective factors. In this section, due to constraints of space, only one sample is presented. After the questionnaire was designed, copies of it were submitted to the 12 industry experts who participated in the study. Next, confirmation and the criteria for analysis were considered. At this stage, the comparison was paired with the relative importance of all the questionnaires. Accordingly, the matrix of paired comparisons was created, and the importance coefficient of the main indicators of performance evaluation, as well as the pairwise matrix of the coefficient of significance of indices relative to the index, were computed .

\subsubsection{Calculation of triangular fuzzy numbers}

Given the relative importance of the calculated values in the previous step, triangular fuzzy numbers were calculated to integrate all expert opinions.

Table 9

Matrix of Triangular Fuzzy Numbers

\begin{tabular}{|c|c|c|c|c|c|c|}
\hline & company strategy & $\begin{array}{l}\text { Manage and improve the } \\
\text { process }\end{array}$ & $\begin{array}{l}\text { Choosing the right } \\
\text { people }\end{array}$ & Focus on customer & $\begin{array}{c}\text { Management } \\
\text { commitment and } \\
\text { resources }\end{array}$ & $\begin{array}{c}\text { Leadership and } \\
\text { Perspectives }\end{array}$ \\
\hline $\begin{array}{l}\text { Leadership and } \\
\text { Perspectives }\end{array}$ & $(1,1,1)$ & $(9,5.614,3)$ & $(1,6.3568,9)$ & $(0.25,8,4.025)$ & $(8,3.949,0.333)$ & $(8,2.039,0.333)$ \\
\hline $\begin{array}{l}\text { Management } \\
\text { commitment and } \\
\text { resources }\end{array}$ & $(0.333,0.178,0.111)$ & $(1,1,1)$ & $(5.0642,0.5,8)$ & $(5,2.506,0.333)$ & $(1,6,2.271)$ & $(0.167,0.779,4)$ \\
\hline Focus on customer & $(0.157,0.111,1)$ & $(0.125,2,0.197)$ & $(1,1,1)$ & $(0.149,0.196,0.5)$ & $(2,0.235,0.125)$ & $(0.111,2,0.202)$ \\
\hline $\begin{array}{l}\text { Choosing the right } \\
\text { people }\end{array}$ & $(4,0.248,0.125)$ & $(3,0.399,0.2)$ & $(8,5.110,2)$ & $(1,1,1)$ & $(1.135,0.333,4)$ & $(7,0.413,0.143)$ \\
\hline $\begin{array}{l}\text { Manage and improve } \\
\text { the process }\end{array}$ & $(3,0.253,0.125)$ & $(1,0.439,0.166)$ & $(4.264,0.5,8)$ & $(0.25,3,0.882)$ & $(1,1,1)$ & $(5,0.466,0.111)$ \\
\hline company strategy & $(3,0.49,0.125)$ & $(6,1.284,0.25)$ & $(9,4.937,0.5)$ & $(7,2.42,0.149)$ & $(9,2.142,0.2)$ & $(1,1,1)$ \\
\hline
\end{tabular}

The triangular fuzzy set was defined via. ãij $=(\alpha \mathrm{ij}, \beta \mathrm{ij}, \delta \mathrm{ij})$ where $\tilde{a}_{i j}$ is the set of triangular fuzzy numbers, $\alpha_{\mathrm{ij}}$ is the least value of criterion $j$ for dimension $i, \beta_{i j}$ is the geometric mean of criterion $j$ for dimension $i$, and $\delta_{i j}$ is the highest criterion value of dimension $j$ for dimension $i$. The information includes the numbers in parentheses, which are respectively least value of criterion $j$ for dimension $i$, 
the geometric mean of criterion $j$ for dimension $i$, and the highest value of criterion $j$ for the dimension $I$ (see Table 9).

\subsection{3. coefficients of each of the pairwise matrices}

At this stage, using the definitions of the fuzzy network analysis process, the coefficients of each of the pairwise matrices were calculated.

$\left[\sum_{i=1}^{m} \sum_{j=1}^{n} M_{i j}\right]=(18.825,64.651,131.833)^{-1}=(0.0075,0.0154,0.0531)$

$S_{1}=(5.916,22.9838,43) *(0.0075,0.0154,0.0531)=(0.4437,0.3539,2.2833)$

$S_{2}=(3.111,11.8182,24.333) *(0.0075,0.0154,0.0531)=(0.02333,0.1820,1.2920)$

$S_{3}=(1.621,1.978,8.5) *(0.0075,0.0154,0.0531)=(0.0121,0.0304,0.4513)$

$S_{4}=(3.801,8.305,27) *(0.0075,0.0154,0.0531)=(0.0285,0.1278,1.4337)$

$S_{5}=(2.152,7.304,21) *(0.0075,0.0154,0.0531)=(0.01614,0.1124,1.1151)$

$S_{6}=(2.224,12.273,35) *(0.0075,0.0154,0.0531)=(0.01668,0.1890,1.8585)$

\subsubsection{The magnitude of each element on other elements}

The magnitude of each element on other elements was calculated (see table 10).

Table 10

The magnitude of each element on other elements was calculated

\begin{tabular}{cccccc}
\hline $\mathrm{S}_{1}$ & $\mathrm{~S}_{2}$ & $\mathrm{~S}_{3}$ & $\mathrm{~S}_{4}$ & $\mathrm{~S}_{5}$ & $\mathrm{~S}_{6}$ \\
\hline$s_{1} \geq s_{2}=1$ & $s_{2} \geq s_{1}=0.8315$ & $s_{3} \geq s_{1}=0.0229$ & $s_{4} \geq s_{1}=0.8140$ & $s_{5} \geq s_{1}=0.7354$ & $s_{6} \geq s_{1}=0.8956$ \\
$s_{1} \geq s_{3}=1$ & $s_{2} \geq s_{3}=1$ & $s_{3} \geq s_{2}=0.7362$ & $s_{4} \geq s_{2}=0.9629$ & $s_{5} \geq s_{2}=0.94$ & $s_{6} \geq s_{2}=1$ \\
$s_{1} \geq s_{4}=1$ & $s_{2} \geq s_{4}=1$ & $s_{3} \geq s_{4}=0.8127$ & $s_{4} \geq s_{3}=1$ & $s_{5} \geq s_{3}=1$ & $s_{6} \geq s_{3}=1$ \\
$s_{1} \geq s_{5}=1$ & $s_{2} \geq s_{5}=1$ & $s_{3} \geq s_{5}=0.8414$ & $s_{4} \geq s_{5}=1$ & $s_{5} \geq s_{4}=0.9862$ & $s_{6} \geq s_{4}=1$ \\
$s_{1} \geq s_{6}=1$ & $s_{2} \geq s_{6}=0.9945$ & $s_{3} \geq s_{6}=0.7326$ & $s_{4} \geq s_{6}=0.9585$ & $s_{5} \geq s_{6}=0.9348$ & $s_{6} \geq s_{5}=1$ \\
\hline
\end{tabular}

After determining the magnitude of each element with respect to other elements, the magnitude of $S_{i}$ items was computed.

$\operatorname{Min} V\left(S_{1} \geq S_{2}, S_{3}, S_{4}, S_{5}, S_{6}\right)=1$

$\operatorname{Min} V\left(S_{2} \geq S_{1}, S_{3}, S_{4}, S_{5}, S_{6}\right)=0.8315$

$\operatorname{Min} V\left(S_{3} \geq S_{1}, S_{2}, S_{4}, S_{5}, S_{6}\right)=0.0229$
$\operatorname{Min} V\left(S_{4} \geq S_{1}, S_{2}, S_{3}, S_{5}, S_{6}\right)=0.8140$

$\operatorname{Min} V\left(S_{5} \geq S_{1}, S_{2}, S_{3}, S_{4}, S_{6}\right)=0.7354$

$\operatorname{Min} V\left(S_{6} \geq S_{1}, S_{2}, S_{3}, S_{4}, S_{5}\right)=0.8956$

These numbers represent the non-normal weights of the indicators $\mathrm{C}_{1}-\mathrm{C}_{6}$, and the following values of the normalized weights are given.

$W=[0.2325,0.1933,0.0053,0.1893,0.1710,0.2083]$

Next the dependence between the criteria calculated through the fuzzy DEMATEL method was considered in this section (see Table 11).

\section{Table 11}

Normalized diaphragmatic relationships matrix

\begin{tabular}{lcccccc}
\hline & $\mathrm{C}_{1}$ & $\mathrm{C}_{2}$ & $\mathrm{C}_{3}$ & $\mathrm{C}_{4}$ & $\mathrm{C}_{5}$ & $\mathrm{C}_{6}$ \\
\hline $\mathrm{C}_{1}$ & 0.15799 & 0.18213 & 0.17987 & 0.18001 & 0.18052 & 0.17953 \\
$\mathrm{C}_{2}$ & 0.16562 & 0.14777 & 0.16884 & 0.16894 & 0.17066 & 0.16968 \\
$\mathrm{C}_{3}$ & 0.17578 & 0.17408 & 0.15160 & 0.17400 & 0.17385 & 0.17220 \\
$\mathrm{C}_{4}$ & 0.14998 & 0.14848 & 0.15268 & 0.13358 & 0.14887 & 0.15456 \\
$\mathrm{C}_{5}$ & 0.17095 & 0.16930 & 0.16691 & 0.16428 & 0.14774 & 0.16817 \\
$\mathrm{C}_{6}$ & 0.17968 & 0.17826 & 0.18010 & 0.17919 & 0.17835 & 0.15585 \\
\hline
\end{tabular}

Criteria were obtained by taking into account dependence by combining the results through the relation $\mathrm{W} \varepsilon=\mathrm{B} \times \mathrm{W}$. 


\begin{tabular}{|c|c|c|c|c|c|c|c|c|c|c|c|}
\hline & $\mathrm{C} 1$ & $\mathrm{C} 2$ & $\mathrm{C} 3$ & $\mathrm{C} 4$ & $\mathrm{C} 5$ & C6 & & & & $\begin{array}{l}\text { Finally } \\
\text { Weight }\end{array}$ & Rank \\
\hline Leadership and perspectives & 0.15799 & 0.18213 & 0.17987 & 0.18001 & 0.18052 & 0.17953 & & 0.2325 & & 0.175692 & 1 \\
\hline Management commitment and resources & 0.16562 & 0.14777 & 0.16884 & 0.16894 & 0.17066 & 0.16968 & & 0.1933 & & 0.165062 & 4 \\
\hline Focus on customer & 0.17578 & 0.17408 & 0.15160 & 0.17400 & 0.17385 & 0.17220 & $x$ & 0.0053 & & 0.169647 & 3 \\
\hline Choosing the right people & 0.14998 & 0.14848 & 0.15268 & 0.13358 & 0.14887 & 0.15456 & & 0.1893 & & 0.150953 & 6 \\
\hline Manage and improve the process & 0.17095 & 0.16930 & 0.16691 & 0.16428 & 0.14774 & 0.16817 & & 0.1710 & & 0.164685 & 5 \\
\hline company strategy & 0.17968 & 0.17826 & 0.18010 & 0.17919 & 0.17835 & 0.15585 & & 0.2083 & & 0.173662 & 2 \\
\hline
\end{tabular}

Table 12 shows the total weights of indicators calculated based on the relationships mentioned above and the processes, along with final weights.

Table 12

Final weights

\begin{tabular}{|c|c|c|c|c|c|c|c|}
\hline Dimension & Weight & Rank & Indicators & $\begin{array}{l}\text { The weight of } \\
\text { the following } \\
\text { component }\end{array}$ & $\begin{array}{c}\text { Rank below } \\
\text { component in } \\
\text { component }\end{array}$ & $\begin{array}{l}\text { The final } \\
\text { weight }\end{array}$ & $\begin{array}{c}\text { The final } \\
\text { rank }\end{array}$ \\
\hline \multirow{12}{*}{ 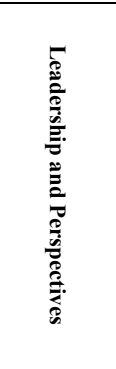 } & \multirow{12}{*}{ 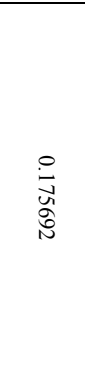 } & \multirow{12}{*}{-} & Providing support & 0.11221 & 1 & 0.0197 & 22 \\
\hline & & & Providing guidance and reward & 0.09312 & 3 & 0.0164 & 26 \\
\hline & & & Empowering employees & 0.08091 & 8 & 0.0142 & 32 \\
\hline & & & $\begin{array}{l}\text { Encouraging employees to focus on important matters and rely on Six Sigma } \\
\text { and the strategic design }\end{array}$ & 0.07098 & 11 & 0.0125 & 35 \\
\hline & & & The perception and acceptance of the senior manager & 0.08212 & 6 & 0.0144 & 30 \\
\hline & & & Making sure of the commitment of chief executive officer & 0.09321 & 2 & 0.0164 & 26 \\
\hline & & & Setting goals at each stage and identifying the responsible employee & 0.09212 & 4 & 0.0162 & 27 \\
\hline & & & Developing an object-oriented approach & 0.08921 & 5 & 0.0157 & 28 \\
\hline & & & Prioritizing customers, shareholders and employees & 0.07676 & 9 & 0.0135 & 33 \\
\hline & & & $\begin{array}{l}\text { Making sure of employees' understanding of the Six Sigma benefit value for } \\
\text { the customer }\end{array}$ & 0.06325 & 12 & 0.0111 & 36 \\
\hline & & & Fostering belief and commitment in employees to attain the prospect & 0.07123 & 10 & 0.0125 & 35 \\
\hline & & & Adopting change & 0.08111 & 7 & 0.0143 & 31 \\
\hline \multirow{7}{*}{ 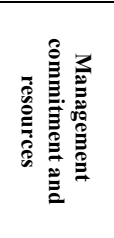 } & \multirow{7}{*}{ 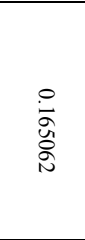 } & \multirow{7}{*}{$\perp$} & Having familiarity with the Six Sigma process & 0.15612 & 3 & 0.0258 & 14 \\
\hline & & & Linking the Six Sigma principles to the goals of the company & 0.14213 & 4 & 0.0235 & 16 \\
\hline & & & $\begin{array}{l}\text { Applying benchmarks to demonstrate the benefits of implementing Six } \\
\text { Sigma projects to shareholders }\end{array}$ & 0.09332 & 7 & 0.0154 & 29 \\
\hline & & & Performing active leadership & 0.12238 & 6 & 0.0202 & 21 \\
\hline & & & Recording continuous improvement of market share (over the last few years) & 0.13123 & 5 & 0.0217 & 19 \\
\hline & & & $\begin{array}{l}\text { Having the ability to cover sufficient financial support for an improvement } \\
\text { project }\end{array}$ & 0.17231 & 2 & 0.0284 & 10 \\
\hline & & & Motivating staff through the HR system & 0.18324 & 1 & 0.0302 & 7 \\
\hline \multirow[b]{2}{*}{ 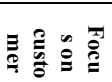 } & \multirow[b]{2}{*}{ ‡: } & \multirow[b]{2}{*}{$\omega$} & Meeting customers' expectations and focusing on them & 0.46123 & 2 & 0.0782 & 2 \\
\hline & & & $\begin{array}{l}\text { Evaluating company's customer orientation, employee development plan, } \\
\text { training programs, job description and annual development plans }\end{array}$ & 0.54009 & 1 & 0.0916 & 1 \\
\hline \multirow{4}{*}{ 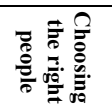 } & \multirow{4}{*}{ 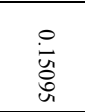 } & \multirow{4}{*}{ a } & Understanding/implementing the Six Sigma logic by project members & 0.12125 & 4 & 0.0183 & 25 \\
\hline & & & Using criteria to select the right people to instruct and implement the project & 0.30793 & 1 & 0.0465 & 3 \\
\hline & & & Assigning flexible/open minded people as project members & 0.29169 & 2 & 0.0440 & 4 \\
\hline & & & Making sure of project members' understanding of Six Sigma tools & 0.27968 & 3 & 0.0422 & 5 \\
\hline \multirow{7}{*}{ 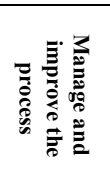 } & \multirow{7}{*}{$\begin{array}{l}\stackrel{0}{\circ} \\
\stackrel{+}{+}\end{array}$} & \multirow{7}{*}{ u } & Using reciprocity for improvement outcomes & 0.18112 & 1 & 0.0298 & 8 \\
\hline & & & Using the SPC tool (process maps, control charts, pareto graphs) & 0.16213 & 4 & 0.0267 & 12 \\
\hline & & & Collecting data for key process functions & 0.11502 & 6 & 0.0189 & 24 \\
\hline & & & Certifying the accuracy of collected data & 0.13518 & 5 & 0.0223 & 17 \\
\hline & & & Keeping costs under control & 0.08123 & 7 & 0.0134 & 34 \\
\hline & & & Investing in employee training and encouraging problem-solving & 0.16216 & 3 & 0.0267 & 12 \\
\hline & & & Assigning a team to solve problems & 0.16324 & 2 & 0.0269 & 11 \\
\hline \multirow{7}{*}{ 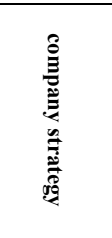 } & \multirow{7}{*}{ 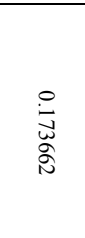 } & \multirow{7}{*}{ N } & Participating in a specifically defined strategy & 0.17812 & 1 & 0.0309 & 6 \\
\hline & & & $\begin{array}{l}\text { Making sure of employees' perception that strategy is the key to company } \\
\text { success }\end{array}$ & 0.15219 & 3 & 0.0264 & 13 \\
\hline & & & Keeping stability in corporate strategy & 0.14421 & 4 & 0.0250 & 15 \\
\hline & & & Devising a well-specified corporate strategy for every member & 0.16428 & 2 & 0.0285 & 9 \\
\hline & & & Considering the improvement process in formulating strategies & 0.12303 & 6 & 0.0214 & 20 \\
\hline & & & $\begin{array}{l}\text { Setting criteria for measuring the extent to which a company's strategy is } \\
\text { achieved }\end{array}$ & 0.11021 & 7 & 0.0191 & 23 \\
\hline & & & Completing small projects before starting larger ones & 0.12812 & 5 & 0.0222 & 18 \\
\hline
\end{tabular}

\section{Conclusion}

This study has investigated the readiness of an Iranian steel industry company for the implementation of Six Sigma projects. Six Sigma is a methodology that helps minimize error in work. It is a statistical measure that enhances accuracy in production, services and processes, while serving as a measurement tool. It also be applied as a business strategy, because it lowers the costs of high quality. The use of the effective and simple techniques proposed in the Six Sigma methodology paves the way for improving the optimal production processes, such as the general process map (SIPOC), defect detection tables, and various measures of process capability measurement, identifying the strengths and weaknesses in organizational processes. This study tried to identify and categorize the factors affecting the implementation and function 
of recovery programs in the steel industry. In addition, contrary to some of the studies focused on barriers, this study included specific notions of improvement such as innovation, pollution reduction, or quality management. Improvement was regarded as a comprehensive concept in this research, as a factor that could reduce time and cost or increase the quality of all projects. Researchers in recent years have frequently relied on the multi-criteria decision-making approach, considering its specific features. Some researchers who tried to categorize the affected components used the analytical tool of multi-criteria decision making, and some have demonstrated that this framework works properly. In this research, attempt was made to provide a conceptual framework for identifying influential factors by examining the elements important for successful implementation of Six Sigma in the organization. Through further statistical analysis, the fuzzy Delphi was used to identify and prioritize the specified indicators (given the high accuracy of fuzzy methods). Moreover, to conduct a better fuzzy analysis of the fuzzy analysis process, the fuzzy DEMATEL method was employed (to specify the interconnection between the indicators). By detecting the interconnection between the indicators, the indicators were prioritized, and the multiple dimensions of the Six Sigma were also taken into account. This study contributes to the line of academic research that examines more precisely the various dimensions of Six Sigma. Broadly speaking, the model proposed in this study has revealed the importance and role of the key elements to Six Sigma success and some of the critical areas that managers need to focus on when implementing the Six Sigma project in an organization. For example, senior management support has a direct impact on the link that Six Sigma can have with human resource management, education, cultural change, and other factors. Therefore, it is imperative that senior management accept Six Sigma concepts for a successful implementation of Six Sigma. Managers should also be willing to allocate sufficient resources to adapt organizational processes, structures and policies to frame and implement the Six Sigma approach. The findings of this study will help develop the knowledge of Six Sigma, as it aims to provide a model for managers and planners of organizations by providing empirical evidence through an analytic model arising from the conditions of Iranian organizations. It is necessary to take into account the circumstances, the amount of resources, and organizational priorities for the compilation and implementation of Six Sigma projects.

In general, the study had some limitations, such as sample size, limited access to steel companies, and perceptional differences exhibited by the respondents in their answers to questionnaire items. These factors should be further controlled to ensure the changeability and consideration of possible external variations for a better validation of the model and the generalizability of the results and the sample size. Researchers interested in this topic can use the following suggestions for further research. they can probe into methods such as in-depth interviews with experts and the theory of diography. Some studies can collect data from manufacturing industries and optimize the proposed model through the data gathered. An interesting topic for further investigation is the factors contributing to the success of Six Sigma in the steel industry and the effect of these factors on various performance dimensions of companies. Another area of search can focus on the effect of other quality management approaches such as ISO on the proposed model.

\section{References}

Antony, J. (2014). Readiness factors for the Lean Six Sigma journey in the higher education sector. International Journal of Productivity and Performance Management, 63(2), 257-264.

Ataei, M. (2010), Fuzzy Multi-criteria Decision Making, First Printing, Shahrood, Shahrood University Press,.

Azar, A., Mir Fakhreddini, H., \& Anvari Rostami, A. A. (2008). A Comparative Study of Data Analysis in Six Sigma, Using Statistical Tools and Multi-Attribute Decision Techniques. Quarterly Journal of Management Researches in Iran, 12(4), 1-35.

Biedry, J. (2001). Linking Six Sigma analysis with human creativity. The Journal for Quality and Participation, 24(4), 36.

Byrne, G., Lubowe, D., \& Blitz, A. (2007). Using a Lean Six Sigma approach to drive innovation. Strategy \& Leadership, 35(2), 5-10.

Chakravorty, S. S. (2009). Six Sigma programs: An implementation model. International journal of production economics, 119(1), 1-16. 
Chandra, A. (2008). Five Six Sigma deployment mistakes and how to avoid them. https://www.isixsigma.com/new-to-six-sigma/getting-started/5-six-sigma-deployment-mistakes-and-howavoid-them/

Chen, M., \& Lyu, J. (2009). A Lean Six-Sigma approach to touch panel quality improvement. Production Planning and Control, 20(5), 445-454.

Cheng, C. H., \& Lin, Y. (2002). Evaluating the best main battle tank using fuzzy decision theory with linguistic criteria evaluation. European journal of operational research, 142(1), 174-186.

Cheng, J. H., Lee, C. M., \& Tang, C. H. (2009). An application of fuzzy Delphi and fuzzy AHP on evaluating wafer supplier in semiconductor industry. WSEAS Transactions on Information Science and Applications, 6(5), 756-767.

El Haouzi, H., Pétin, J. F., \& Thomas, A. (2009). Design and validation of a product-driven control system based on a six sigma methodology and discrete event simulation. Production Planning and Control, 20(6), 510524.

Henderson, K. M., \& Evans, J. R. (2000). Successful implementation of six sigma: benchmarking General Electric Company. Benchmarking: An International Journal, 7(4), 260-282.

Hensley, R. L., \& Dobie, K. (2005). Assessing readiness for six sigma in a service setting. Managing Service Quality: An International Journal, 15(1), 82-101.

Jassbi, J., Mohammad nejad, F., \& Nasrollahzadeh, H. (2010). A Fuzzy Dematel framework for modeling cause and effect relationship of trategy, Expert systems with applications, 38 (5),5967-5973.

Kazazi, A., \& Saroukhani, D. (2005). Identification of key factors in the effective use of Six Sigma programs in different organizations (large multinational, small and medium sized organizations, services). Third International Management Conference.

Khobyari, B. (2003). Six Sigma and its role in improving the efficiency and effectiveness of the organization's processes. Organization Development, 50, 20-27.

Krueger, D. C., Mellat Parast, M., \& Adams, S. (2014). Six Sigma implementation: a qualitative case study using grounded theory. Production Planning \& Control, 25(10), 873-889.

Lagrosen, Y., Chebl, R., \& Rios Tuesta, M. (2011). Organisational learning and Six Sigma deployment readiness evaluation: a case study. International Journal of Lean Six Sigma, 2(1), 23-40.

Lee, T. Y., Wong, W. K., \& Yeung, K. W. (2011). Developing a readiness self-assessment model (RSM) for Six Sigma for China enterprises. International Journal of Quality \& Reliability Management, 28(2), 169194.

Liu, Y. C., \& Chen, C. S. (2007). A new approach for application of rock mass classification on rock slope stability assessment. Engineering geology, 89(1), 129-143.

NikFarjam, R. \& Norosana, R. (2009). Identification of Key Factors in the Implementation of Six Sigma Projects at Sarcheshmeh Copper Complex. Seventh International Management Conference.

Norosana, R., Saghaee, A., \& Amiri, A. H. (2013). Design for Six Sigma. Tehran. Shahed University.

Rajkumar, S. (2014). Impact of implementing Six-Sigma on the workplace efficiency in service industries in Karnataka. Asia Pacific Journal of Research Vol: I Issue XIII, 5-13.

Reyes, F., Cerpa, N., Candia-Véjar, A., \& Bardeen, M. (2011). The optimization of success probability for software projects using genetic algorithms. Journal of Systems and Software, 84(5), 775-785.

Saaty, T. L. (2004). Decision making - the analytic hierarchy and network processes (AHP/ANP). Journal of systems science and systems engineering, 13(1), 1-35.

Salehi Sedghiani, J., \& Roustai, A. (2005). Six Sigma Position in Improving Customer Satisfaction. Accountant Journal, 20 (168), 26-29.

Snee, R. D. (2000). Impact of Six Sigma on quality engineering. Quality Engineering, 12(3), 9-14.

Sodhi, M. S., \& Sodhi, N. S. (2005). Six sigma pricing. Harvard Business Review, 83(5), 135-142.

Zgardy, S. H., Bagheri, S., \& Attaryan, J. (2010). Statistical Analysis of Relationships Between the Key Factors of Successful Six Sigma Projects in Selected Iranian Companies. Journal of Industrial Engineering, 44 (1), 49-62.

Zhou, Q., Huang, W., \& Zhang, Y. (2011). Identifying critical success factors in emergency management using a fuzzy DEMATEL method. Safety science, 49(2), 243-252.

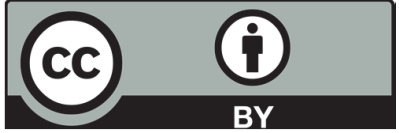

(C) 2018 by the authors; licensee Growing Science, Canada. This is an open access article distributed under the terms and conditions of the Creative Commons Attribution (CC-BY) license (http://creativecommons.org/licenses/by/4.0/). 